\title{
Emerging applications of flow cytometry for clinical microbiology
}

\section{Alexandra Mihaela Velican ${ }^{1}$, Luminița Maruțescu ${ }^{1 凶}$, Claude Lambert $^{3}$, Mariana Carmen Chifiriuc ${ }^{1,2}$}

${ }^{1}$ Research Institute of the University of Bucharest (ICUB), Bucharest, Romania; ${ }^{2}$ Department of Microbiology and Immunology, Faculty of Biology, University of Bucharest, Romania; ${ }^{3}$ L'unité de cytométrie en flux du CHU de SaintEtienne, France

${ }^{\square}$ Correspondence to: Luminița Maruțescu, Department of Microbiology and Immunology, Faculty of Biology, University of Bucharest, Romania; E-mail: lumi.marutescu@gmail.com

Received: 29 March 2019 / Revised: 18 July 2019 / Accepted: 29 July 2019 / Available online: 3 August 2019

\begin{abstract}
During the last years, considerable effort has been devoted to the development of rapid and sensitive methods for microbial pathogens detection and antibiotic susceptibility assay. Flow cytometry (FCM) is an excellent tool that could provide both speed and specificity for the clinical microbiology. FCM can be applied directly on the clinical sample, bypassing the need for microbiological cultures and enabling earlier clinical decision. This could both improve the emergency treatment of infected patients and avoid the treatment in case of negative samples. This minireview briefly describes the current applications of FCM in clinical microbiology, for the detection and enumeration of microbial pathogens (differentiation of Gram-positive and Gram-negative bacteria using fluorescent nucleic acid binding dyes, investigation of bacteriuria), study of microbial physiology, assessment of antimicrobial susceptibility (both for bacterial and fungal strains), establishing drug synergies and investigating the resistance mechanisms.
\end{abstract}

Keywords: flow cytometry, microbial cells detection, antimicrobial susceptibility, antibiotic resistance

\section{Introduction}

Despite vaccination and antibiotic treatment availability, microbial infections still represent one of the most important global threats for the public health. The rapid and early detection of infected patients represent an essential step both for the treatment as well as for the epidemiological control of infectious diseases; consequently, one of the most important research directions is represented by the development of rapid, specific and sensitive methods for clinical microbiology laboratories. In this purpose, in the recent years, microbiological techniques have been increasingly complemented by technologies which provide speed and specificity to microbiological diagnosis, including flow cytometry (FCM) (Pieretti et al., 2012). By the aid of its fluid, optical and electronic systems, FCM allows suspended particles/cells circulating in a laminar flow to be interrogated one by one with a laser beam. The collected optical signals resulting from the scattered light and/or from the fluorescence of the particle are converted in electronic signals, which are further collected for data acquisition and analysis through photodetectors (photodiodes and photomultiplier tubes) (Adan et al., 2017).
FCM is an excellent tool, still insufficiently explored for the clinical microbiology, allowing the study of microbial activity and microbial cells quantification, as well as of antimicrobial drugs' activity, synergy and mechanisms of resistance (Pina-Vaz et al., 2005; Pina-Vaz et al., 2010; Teixeira-Santos et al., 2012; Faria-Ramos et al., 2012). This minireview briefly describes the current applications of FCM in clinical microbiology, for the microbial detection and enumeration of microbial pathogens and for the assessment of antimicrobial susceptibility and resistance mechanisms.

\section{Using FCM for microbial detection and identification in clinical laboratories}

Currently, FCM protocols have been developed for the differentiation of Gram-positive and Gram-negative bacteria, which are being used for the detection of bacterial cells directly in clinical specimens or in liquid cultures. An alternative Gram staining was optimized, using a combination of two fluorescent nucleic acid binding dyes, i.e. hexidium iodide (HI), entering only in Gram-positive and SYTO 13, entering in both Grampositive and Gram-negative cells. 
When the dyes are used in combination, the Gramnegative cells will have a green fluorescence, given by SYTO 13, while the Gram-positive cells will have a redorange one, due to HI (Mason et al., 1998). Using FCM to interrogate the cells colored with the combination of two fluorescent nucleic acid binding dyes, it correctly predicted the Gram affinity of 45 Gram-positive, Gramnegative or Gram-variable clinical strains. Moreover, some Gram-positive anaerobic strains which were considered Gram-negative by using the standard Gram staining were correctly classified using the fluorescent Gram stain (Mason et al., 1998).

Urinary tract infections (UTIs) are the second most common bacterial infections. The current gold standard method for the diagnosis of UTIs is based on the analysis of urine culture that requires 18-48 hours for the identification of the etiological agents and additional 24 hours until the results of antimicrobial susceptibility testing are available. It has been shown that FCM could easily discriminate between Gram-positive and Gramnegative bacteria in liquid culture media within $10 \mathrm{~min}$, proving thus to be an excellent tool for the rapid diagnosis and guiding of a targeted antibiotic treatment in UTI patients, especially for those experiencing acute episodes (Wada et al., 2012). FCM-based devices were also used for establishing specific FCM profiles of urine samples with bacteriuria, leukocyturia, and erythrocyturia, collected from patients with a suspected UTI and to correlate these aspects with the results of standard urine microscopic examination and culture (Monsen and Ryden, 2015). Therefore, the automatic FCM based systems could therefore be used to confirm UTI, define the antibiotic resistance profile of uropathogens and avoid processing negative urine cultures (Okada et al., 2000a; Okada et al., 2000b; Okada et al., 2006; Pieretti et al., 2012; Moshaver et al., 2016).

In order to shorten the time of urine sample processing, a combinatin of both FCM and matrix-assisted laser desorption/ionization time-of-flight mass spectrometry (MALDI-TOF-MS) was used for microbial uropathogens screening and identification, respectively. The total duration of the optimized protocol used to diagnose UTI was about 1 hour for microbial identification and of 1824 hours for the antimicrobial susceptibility testing (Zboromyrska et al., 2016).

Fluorescence in situ hybridization (FISH) using nucleic acid probes and FCM have been used together as a possible approach for the identification of Staphylococus sp. directly in blood cultures (Hartmann et al., 2005). Candida albicans and Enterobacteriaceae strains could be also directly detected in blood culture bottles using FCM-FISH, using the universal yeast probe PF-2 (green) and a specific probe Calb-1249 (red) for C. albicans, while for Enterobacteriaceae, the universal bacterial probe Eub-338 (green) and a specific probe Ent-186 (red) were used. The results demonstrate the potential of FCFISH to be used for detection and identification of Gramnegative rods and yeasts directly from blood cultures, thus helping to improve the outcome of septicemic patients (Kempf et al., 2005).

Protein A expression was a reliable parameter for $S$. aureus FCM identification in clinical samples. In liquid cultures obtained from throat, nose, groin and wounds, $S$. aureus cells could be clearly distinguished from debris on the basis of scattered signals after $3-5$ hours incubation, with a specificity of $96.1 \%$ and a sensitivity of $97.5 \%$ (McMullan et al., 2015).

FCM seems to be a promising strategy for the rapid detection of viable Cryptococcus neoformans, one of the most frequent causes of fungal meningitis, which is responsible of significant mortality rates in immunodepressed patients, despite appropriate therapy. The quantitative assessment of viable cryptococci in the cerebrospinal fluid during antifungal therapy has been shown to have both prognostic and therapeutic value. The quantitative microscopy with exclusion staining using trypan blue dye, and the FCM using the fluorescent dye 2 -7 -Bis- (2-carboxyethyl)-5-(6)-carboxyfluorescein, acetoxymethyl ester (BCECF-AM) have been proved to be practicable and faster alternatives to the standard methods used for the quantitative assessment of viable fungal cells (Nuding and Zabel, 2013).

\section{Assessement of antimicrobial suseptibility and resistance}

Currently, antibiotic resistance is considered on of the top priorities for the public health at global level. There are many factors responsible for the acceleration of the natural process of bacterial resistance, including the rapid adaptation of bacterial cells to the selective pressure exerted by antibiotics sustained by a huge diversity and mobility of resistance genes, over prescription of antibiotics and self medication (Fair and Tor, 2014). Therefore, developing rapid, accurate, sensitive and costeffective methods that can quickly detect: (1) if a certain strain is resistant to antibiotics, (2) the susceptibility to certain antimcirobials and (3) the appropriate dosage required for a particular infectious agent represents one of the research priorities for the biomedical field (Kaittanis et al., 2008). The traditional methods frequently used for the detection of antibiotic resistance in clinical settings are generally slow requiring previous isolation and identification. There have been proposed faster methods, such as those based on PCR detection of antibiotic resistance genes, but they are not always predictive for the efficiency of a certain antibiotic, particularly in the cases in which resistance is not due to the presence of resistance genes, being the consequence, for example, of biofilm development. Therefore, alternative methods, such as FCM-based detection of bacterial physiology changes caused by antibiotics are being currently explored (Saint-Ruf et al., 2016). New fluorescent dyes indicating the presence of metabolic changes induced by antibiotics are investigated to 
improve the reliability of FCM antibiotic susceptibility testing (Saint-Ruf et al., 2016).

However, many optimization steps are needed, to face challenges such as the dependence of fluorescence signals on microbial cells size, or the chemical and metabolic heterogeneity of bacterial species and populations under suboptimal culture conditions (such as antibiotic exposure) (Shapiro, 2001). The parameters employed in the FCM assays for the evaluation of bacterial physiology changes in the presence of antibiotics are: increased carbonylation of cellular macromolecules induced by antibiotics (Saint-Ruf et al., 2016), membrane integrity loss that could be detected by using propidium iodide (PI) or TO-PRO®-3 (Braga et al., 2013; Kerstens et al., 2014); membrane potential loss that could be detected by using $\operatorname{DiBAC}_{4}(3)$ (Bis-(1,3Dibutylbarbituric acid) (Suller et al., 1997; Joux and Lebaron 2000) and the alterations of forward scatter and side scatter light, indicating changes in the cellular size and protein content. Investigations have indicated that Alexa Fluor 633 Hydrazide (AFH), targeting the carbonyl group and TO-PRO R-3 dyes are more appropriate than $\operatorname{DiBAC}_{4}(3)$ for antibiotic susceptibility testing by FCM.

The membrane potential specific agent DiBAC4(3) was used by Nuding et al. to develop a rapid antibiotic susceptibility assay (Nuding et al., 2006; Nuding et al., 2009), that could be used for a wide range of bacterial species. $\mathrm{DiBAC}_{4}(3)$ was used in a FCM assay to examine the membrane integrity loss induced by benzylpenicillin, methicillin and vancomycin in five methicillin-resistant $S$. aureus (MRSA) clinical isolates and in two methicillin-susceptible Staphylococcus aureus (MSSA) reference strains. An enhanced DiBAC4(3) uptake was revealed after treatment with different vancomycin concentrations (i.e. 0.1, 1, 4 and $10 \mathrm{x}$ MIC), the FCM results being in agreement with the standard viable cell counts method performed on agar media (Suller et al., 1997). The antibiotic susceptibility patterns to benzylpenicillin, methicillin and vancomycin for all isolates used in this study could be determined by FCM in 2-4 hours from an overnight plate culture, unlike the standard assay, which needs 18-24 hours for a result. The FCM assay could differentiate between MRSA and MSSA after 2 hours of incubation in oxacillin-containing liquid medium, based on the changes in the transmembrane electrochemical potential induced by antibiotic (Shrestha et al., 2011).

FCM has been also used to detect the effects of macrolides (rokitamycin and erythromycin) on the viability of the Streptococcus pyogenes with mucous phenotype, being able to rapidly distinguish between susceptible and resistant strains (Braga et al., 2013).

An adapted version of the DiBAC4(3) -based FCM assay proposed by Nuding and Zabel, 2013 was used to rapidly detect the extended spectrum beta lactamase (ESBL) producing bacterial strains directly from clinical samples, such as blood cultures or swabs. Simultaneous analysis of the oxacillin susceptibility also allowed the MRSA detection.

The FCM assay of antibiotic susceptibility using AFH dye proved to measure more accurately the minimal inhibitory concentrations (MIC) of different antibiotics against Escherichia coli and Pseudomonas aeruginosa strains, which have been proved to be different, although the respective strains have been previously considered to have the same MIC, calculated by using the standard microdilution assay (Saint-Ruf et al., 2016). However, it must be taken into account that the treatment of $E$. coli cells with different bactericidal antibiotics could give false-positive, background signals caused by an increased autofluorescence, that could mask any specific effects produced by antibiotics, such as the changes in reactive oxygen species release profile (Renggli et al., 2013).

SYTO 16 dye has been successfully used for determining the susceptibility of Mycobacterium tuberculosis to streptomycin, isoniazid, rifampicin and ethambutol (SIRE). After 72 hours of incubation, the FCM assay revealed a clear distinction between the sensitive, intermediate and resistance susceptibility phenotypes (Pina-Vaz et al., 2005).

FCM and computational analysis were used for the investigation of carbapenems combinations synergic activity against Enterobacteriaceae strains producing different carbapenemases and the obtained results showed that the synergistic effect could be detected and quantified even at a low antibiotic concentration and after a short incubation time (Pina-Vaz et al., 2016).

The shortest time achieved by using FCM for the microbial identification and antibiotic susceptibility testing directly from positive blood cultures was reported by March et al. (2015) to be of 2 hours for Gram-negative bacteria and enterococci, and of 3 hours for staphylococci. The FCM protocol used blood culture bottles artificially spiked with different strains and incubated in the presence of different concentrations of vancomycin, oxacillin, ampicillin, ceftazidime, cefotaxime, amikacin, colistin and ciprofloxacin. The results were read by a Sysmex UF-1000i flow cytometer, which is available in the clinical laboratories for urine screening. For method validation, a number of 100 positive monobacterial blood culture bottles from patients hospitalized were processed; the results of direct identification and susceptibility testing from positive blood culture bottles were $100 \%$ in agreement with the results obtained with commercial cultivation-based systems (VITEK 2, MicroScan and E-test) (March et al., 2015).

FCM could also be used for assessing antifungal susceptibility, since it can detect different physiological behaviours induced by different antifungal agents by using appropriate fluorescent markers (Chaturvedi et al., 2004; Czechowska et al., 2008). FCM protocols for assessing susceptibility to azoles, amphotericin B, and echinocandins have already been described. Ramani et al., 2003 evaluated the susceptibility of Aspergillus 
fumigatus isolates to voriconazole, amphotericin B and itraconazole by FCM analysis. The FCM results were obtained within 3 - 4 hours after antifungal treatment and were in good agreement with the MIC values obtained by the standard broth microdilution method. Using two fluorescent dyes, i.e. FUN-1 and PI, Pina-Vaz et al. could assess in dynamics the changes in the behavior of yeast cells upon incubation with serial voriconazole, itraconazole and caspofungin drug concentrations, in order to establish the susceptibility profiles of Candida spp. and Cryptococcus neoformans to the three drugs used (Pina-Vaz et al., 2001). The FUN-1 dye proved to be better than PI, regarding the accuracy of the susceptibility results (Ramani et al, 1997). The FCM method using acridine-orange as a viability marker was also used to assess fungal susceptibility to fluconazole and echinocandin (Pina-Vaz et al., 2001; Rudensky et al., 2005). Susceptibility results were obtained within 5 hours or less, and were in agreement with those obtained by using the standard MIC assay, regarding the sensitivity and specificity. Recently, a FCM protocol using two fluorescent dyes, i.e. FUN-1 and $\mathrm{DiBAC}_{4}(3)$ was used to evaluate the synergic effect of the anidulafungin + amphotericin B and respectively anidulafungin + azoles (fluconazole and voriconazole) combinations against Candida spp. strains. The obtained results were in perfect agreement with those obtained by using the viable cell counts method (Teixeira-Santos et al., 2012).

FCM can also be employed to monitor the effect of different substances on microbial cells, which opens up interesting possibilities for screening the efficiency of novel antimicrobial strategies (Gant et al, 1993; Walberg et al., 1996; Jepras et al., 1997; Roth et al., 1997; Kirk et al., 1998; Mortimer et al., 2000; Novo et al., 2000; Alvarez-Barrientos et al. 2000; Gauthier et al., 2002).

\section{Conclusion}

FCM is a great tool that is already used in ecology and industry to rapidly detect and to enumerate specific microbial cells. However, FCM applications are still under-explored in the field of clinical microbiology. In the current context of the global threath of antimicrobial resistance, the FCM-based methods can represent promising alternatives for the rapid and accurate detection of resistant bacteria, for the assessment of antimicrobial susceptibility and for investigating the resistance mechnisms. The technological advancements will make possible the miniaturisation and automation of FCM devices, allowing to revolutionize their applications in the near future.

\section{Acknowledgments}

The financial support of the project PN-III-P4-ID-PCE-20160921 - Rapid flow cytometric method for microbial detection and antibiotic susceptibility assay directly from clinical specimens is gratefully acknowledged.
CThe Author(s) 2019

Open Access This article is distributed under the terms of the Creative Commons Attribution 4.0 International License (http://creativecommons.org/licenses/by/4.0/) which permits unrestricted use, distribution, and reproduction in any medium, provided you give appropriate credit to the original author(s) and the source, provide a link to the Creative Commons license, and indicate if changes were made.

\section{References}

Adan A., Alizada G., Kiraz Y., Baran Y., Nalbant A. 2017. Flow cytometry: basic principles and applications. Crit Rev Biotechnol. 37, 163-176.

Alvarez-Barrientos A., Arroyo J., Cantòn R., Nombela C., Sànchez-Pérez M, 2000. Applications of Flow Cytometry to Clinical Microbiology. Clin Microbiol Rev. 13, 167-195.

Braga P.C., Bovio C., Culici M., Dal Sasso M. 2013. Flow cytometric assessment of susceptibilities of Streptococcus pyogenes to erythromycin and rokitamycin. Antimicrob. Agents Chemother. 47, 408-412.

Chaturvedi V., Ramani R., Raller M.A. 2004. Collaborative study of the NCCLS and flow cytometry methods for antifungal susceptibility testing of Candida albicans. J. Clin. Microbiol. 42, 2249-2251,

Czechowska K., Johnson D.R., van der Meer J.R. 2008. Use of flow cytometric methods for single-cell analysis in environmental microbiology. Curr. Opin. Microbiol. 11, 205-212.

Fair R.J., Tor Y. 2014. Antibiotics and bacterial resistance in the 21st century. Perspect. Medicin. Chem. 6, 25-64.

Faria-Ramos I., Espinar M.J., Rocha R., Santos-Antunes J., Rodrigues A.G., Canto R., Pina-Vaz C. 2012. A novel flow cytometric assay for rapid detection of extended-spectrum beta-lactamases. Clin. Microbiol Infect. 19, E8-E15.

Gant V.A., Warnes G., Phillips I., Savidge G.F. 1993. The application of flow cytometry to the study of bacterial responses to antibiotics. J. Med. Microbiol. $39,147-154$.

Gauthier C., St-Pierre Y., Villemur R. 2002. Rapid antimicrobial susceptibility testing of urinary tract isolates and samples by flow cytometry. J Med. Microbiol. 51, 192-200.

Hartmann H., Stender H., Schäfer A., Autenrieth I.B., Kempf V.A.J. 2005. Rapid Identification of Staphylococcus aureus in blood cultures by a combination of fluorescence In Situ hybridization using peptide nucleic acid probes and flow cytometry. J. Clin.Microbiol. 43, 4855-4857.

Jepras R.I., Paul F.E., Pearson S.C., Wilkinson M.J. 1997. Rapid assessment of antibiotic effects on Escherichia coli by bis-(1,3-dibutylbarbituric acid) 
trimethine oxonol and flow cytometry. Antimicrob. Agents Chemother. 41, 2001-2005

Joux F., Lebaron P. 2000. Use of fluorescent probes to assess physiological functions of bacteria at singlecell level. Microbes Infect. 2, 1523-1535.

Kaittanis C., Nath S., Perez J.M. 2008. Rapid Nanoparticle-Mediated Monitoring of Bacterial Metabolic Activity and Assessment of Antimicrobial Susceptibility in Blood with Magnetic Relaxation. PLos One 3, e3253.

Kempf,V.A.J., Mändle T., Schumacher U., Schäfer A., Autenrieth I.B. 2005. Rapid detection and identification of pathogens in blood cultures by fluorescence in situ hybridization and flow cytometry. Int. J. Med. Microbiol. 295, 47-55.

Kerstens M., Boulet G., Tritsman C., Horemans T., Hellings M., Delputte P. 2014. Flow cytometric enumeration of bacteria using TO-PRO(R)-3 iodide as a single-stain viability dye. J. Lab. Autom. 19, 555561.

Kirk S.M., Schell R.F., Moore A.V. Callister SM, Mazurek G.H. 1998. Flow cytometric testing of susceptibilities of Mycobacterium tuberculosis isolates to ethambutol, isoniazid, and rifampin in 24 hours. J Clin Microbiol. 36, 1568-1573.

March G.A., García-Loygorri M.C., Simarro M., Gutiérrez M.P., Orduña A., Bratos M.A. 2015. A new approach to determine the susceptibility of bacteria to antibiotics directly from positive blood culture bottles in two hours. J. Microbiol. Methods 109, 49-55.

Mason D.J., Shanmuganathan S., Mortimer F.C., Gant V.A. 1998. A fluorescent Gram stain for flow cytometry and epifluorescence microscopy. Appl. Environ. Microbiol. 64, 2681-2685.

McMullan B.J., Desmarini D., Djordjevic J.T., Chen S.A., Roper M., Sorrell T.C. 2015. Rapid microscopy and use of vital dyes: potential to determine viability of Cryptococcus neoformans in the clinical laboratory. Plos One 10, e0117186.

Monsen T., Ryden P. 2015. Flow cytometry analysis using Sysmex UF-1000i classifies uropathogens based on bacterial, leukocyte, and erythrocyte counts in urine specimens amoung patients with urinary tract infection. J. Clin. Microbiol. 53, 539-545.

Mortimer F.C., Mason D.J., Gant V.A. 2000. Flow cytometric monitoring of antibiotic-induced injury in Escherichia coli using cell-impermeant fluorescent probes. Antimicrob. Agents Chemother. 44, 676-681.

Moshaver B., Boer F., Egmond-Kreileman H., Kramer E., Stegem E., Groeneveld P. 2016. Fast and accurate prediction of positive and negative urine cultures by flow cytometry. BMC Infect Dis. 16, 1-7.

Novo D.J., Perlmutter N.G., Hunt R.H., Shapiro H.M. 2000. Multiparameter flow cytometric analysis of antibiotic effects on membrane potential, membrane permeability, and bacterial counts of Staphylococcus aureus and Micrococcus luteus. Antimicrob Agents Chemother. 44, 827-834.

Nuding S., Fellermann K., Wehkamp J., Mueller H.A., Stange E.F. 2006. A flow cytometric assay to monitor antimicrobial activity of defensins and cationic tissue extracts. J Microbiol Methods 65, 335-345.

Nuding S., Zabel L.T, Enders C., Porter E., Fellermann K. 2009. Antibacterial activity of human defensins on anaerobic intestinal bacterial species: a major role of HBD-3. Microbes Infect. 11, 384-393.

Nuding S., Zabel L.T. 2013. Detection, identification, and susceptibility testing of bacteria by flow cytometry. J Bacteriol. Parasitol. S5-005.

Okada H., Sakai Y., Miyazaki S., Arakawa S., Hamaguchi Y., Kamidono S. 2000a. Detection of significant bacteriuria by automated urinalysis using flow cytometry. J. Clin. Microbiol. 38, 2870-2872.

Okada H., Sakai Y., Kawabata G., Fujisawa M., Arakawa S., Hamaguchi Y., Kamidono S. 2000b. Automated urinalysis. Evaluation of the Sysmex UF-50. Am. J. Clin. Pathol. 115, 605-610.

Okada H., Shirakawa T., Gotoh A., Kamiyama Y., Muto S., Ide H., Hamaguchi A., Horie S. 2006. Enumeration of bacteria cells number and detection of significant bacteriuria use of a new flow cytometrybased device. J. Clin. Microbiol. 44, 3596-3599.

Pieretti B., Masucci A., Moretti M. 2012. Applications of Flow Cytometry to clinical microbiology, Clinical flow cytometry - emerging applications. Schmid I. (Ed.), Intech.

Pina-Vaz C., Sansonetty F., Rodriguez A.G., CostaOliveira S., Tavares C., Martinez-de-Oliveira J. 2001. Cytometric approach for a rapid evaluation of susceptibility of Candida strains to antifungals. Clin Microbiol Infect. 7, 609-618.

Pina-Vaz C., Costa-de-Oliveira S., Rodrigues A.G. 2005. Safe susceptibility testing of Mycobacterium tuberculosis by flow cytometry with the fluorescent nucleic acid stain SYTO 16. J Med. Microbiol. 54, 7781.

Pina-Vaz C., Rodrigues A.G. 2010. Evaluation of antifungal susceptibility using flow cytometry. Methods Mol Biol. 63, 281-289.

Pina-Vaz C., Silva A.P., Faria-Ramos I., Teixeira-Santos R., Moura D., Vieira T.F., Sousa S.F., Costa-deOliveira S., Cantón R., Rodrigues A.G. 2016. A Flow Cytometric and Computational Approaches to Carbapenems Affinity to the Different Types of Carbapenemases. Front. Microbiol. 7, 1-6.

Ramani R., Ramani A., Wong S.J. 1997. Rapid Flow Cytometric Susceptibility Testing of Candida albicans. J. Clin. Microbiol. 35, 2320-2324.

Ramani R. Gangwar M., Chaturvedi V. 2003. Flow cytometry antifungal Susceptibility testing of Aspergillus fumigatus and comparison of mode of action of voriconazole vis-a'-vis amphotericin B and itraconazole. Antimicrob. Agents Chemother. 47, 3627-3629. 
Renggli S., Keck W., Jenal U., Ritza D. 2013. Role of autofluorescence in flow cytometric analysis of Escherichia coli treated with bactericidal antibiotics. J. Bacteriol. 195, 4067-4073.

Roth B.L., Poot M., Yue S.T., Millard P.J. 1997. Bacterial viability and antibiotic susceptibility testing with SYTOX Green nucleic acid stain. Appl. Environ. Microbiol. 63, 2421-2431.

Rudensky B., Broidie E., Yinnon A.M., Weitzman T., Paz E., Keller N., Raveh D. 2005. Rapid flowcytometric susceptibility testing of Candida species. J. Antimicrob. Chemother. 55, 106-109.

Saint-Ruf C., Crussard S., Franceschi C., Orenga S., Ouattara J., Ramjeet M., Surre J, Matic I, 2016, Antibiotic susceptibility testing of the Gram-Negative bacteria based on flow cytometry. Front. Microbiol. 7 , 1121-1129.

Shapiro H.M. 2001. Multiparameter flow cytometry of bacteria: implications for diagnostics and therapeutics. Cytometry 43, 223-226.

Shrestha N.K., Scalera N.M., Wilson D.A., Procop G.W. 2011. Rapid differentiation of Methicillin-Resistant and Methicillin-Susceptible Staphylococcus aureus by flow cytometry after brief antibiotic exposure. J Clin Microbiol. 49, 2116-2120.
Suller M.T., Starkb J.M., Lloyda D. 1997. A flow cytometric study of antibiotic-induced damage and evaluation as a rapid antibiotic susceptibility test for methicillin-resistant Staphylococcus aureus. J Antimicrob. Chemother. 40, 77-83.

Teixeira-Santos R., Rocha R., Moreira-Rosário A., Monteiro-Soares M., Cantón E., Rodrigues A.G., Pina-Vaz C. 2012. Novel Method for Evaluating In Vitro activity of anidulafungin in combination with amphotericin B or azoles. J. Clin. Microbiol. 50, 2748-2754.

Wada A., Kono M., Kawauchi S., Takagi Y., Morikawa T. 2012. Rapid Discrimination of Gram-Positive and Gram-Negative Bacteria in Liquid Samples by Using $\mathrm{NaOH}-$ Sodium Dodecyl Sulfate Solution and Flow Cytometry. Plos One 7, 1-10.

Walberg M., Gaustad P., Steen H.B. 1996. Rapid flow cytometric assessment of mecillinam and ampicillin bacterial susceptibility. J. Antimicrob. Chemother. 37, 1063-1075.

Zboromyrska Y., Rubio E., Alejo A., Vergara A., Mons I., Campos J., Bosch J., Marco F., Vila J. 2016. Development of a new protocol for rapid bacterial identification and susceptibility testing directly from urine samples. Clin. Microbiol Infect. 22, e1 - e6. 